\title{
Reincarnate Historic Systems On FPGA with Novel Design Methodology
}

\author{
Naohiko Shimizu \\ IP ARCH, Inc. Tokai University \\ http://www.ip-arch.jp
}

\begin{abstract}
In this paper, I introduce my and my students projects to reincarnate historic systems on FPGA. Our projects are not replica nor paper-model of historic systems, but reorganized and working system on FPGA with novel and progressive design methodology. I mean progressive as under the development, because I have developed them and I am still improving the methodology and tools very often to use them by myself.

In this paper, I also introduce my design methodology and tools which is used in my and my students projects.
\end{abstract}

\section{INTRODUCTION}

I love computer. I love CPU. After 10 years experience on mainframe and super computers design at Hitachi, I quited Hitachi and move to Tokai University in 1995. I have two reasons, one is that Hitachi decided to stop development of mainframe CPU, another is that I believe the future of FPGA for MY systems.

When I became a teacher, I hoped students to learn computer system from the history. In Japanese language, the origin of the word 'Manabu' which means 'study' is 'Maneru' which means 'copy.' Artists start their life with copy of good styles and techniques. Many technicians in traditional art also start their life with copy of the master's style.

On the other hand, with engineering curriculum, we will start to study fundamental theories in comprehensive subjects. We use projects and laboratory experience to compensate for the gap between fundamental theories and engineering style[1]. But project sometimes is too small to get knowledge from them. And the laboratory experience sometimes is too difficult for undergraduate students to learn the engineering style or design from it.
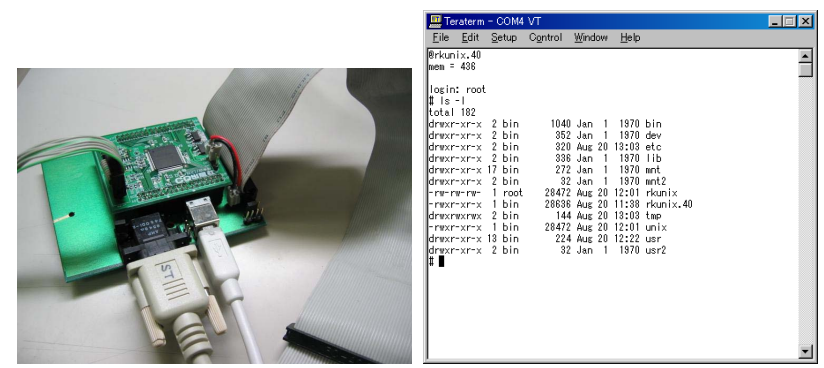

Fig. 1. PDP11/40m compatible system on an FPGA (Altera Cyclone EP1C3) and the console output when running the UNIX v6

I believe case study on existing or historical system is one of a good choice for undergraduate project. In this purpose, the system must be consist of hardware and software. Then students will learn the relationship or co-operation of them. But case studies on real systems are a little difficult, because many state of art systems will not describe their detailed engineering implementation design and methods in publications.

In this paper, I will introduce some of our past projects and design methodology to make up for that with historic systems.

\section{OUR PAST PROJECTS OVERVIEW}

The target systems for the projects were listed as:

2000 CP/M80 system with solid state storage[2].

2001 PCI bus controller[3].

2002 PDP11/40m compatible system with UNIX running on it[4][5].

2002 Space Invaders[6].

2003 FreeDOS system[7].

2004 Vax11 compatible CPU without MMU[8].

2005 Hard wired painting tool[9].

2007 UML based 6502 CPU development[10].

2009 UML based MIPS subset CPU development[11].

I assigned two student for Space Invaders, three for painting tool and four for UML based 6502. But most of the projects are carried out with only one undergraduate student.

We mainly use free tools because most students need extra work at their home in mid night. And they need to run tools on their own PC. Free EDA tools for the projects are:

1995 Alliance VHDL[12]

1996- PARTHENON[13]

2002- sfl2vl[14], Icarus Verilog[15]

2009- above plus uml2sfl

From 1996, I changed the hardware description language to SFL with PARTHENON EDA system. Because many students had difficulty with VHDL, and the combination of PARTHENON and SFL makes their life easy. But the license of PARTHENON is restricted to educational use only. If graduated students want to use SFL, there was no option. Then I wrote a new synthesizer 'sfl2vl' in 2002, and started up IP ARCH, Inc. to provide SFL for industry. The sfl2vl converts SFL file to VerilogHDL. At these days, I think it is important for students to have some experience on VerilogHDL. With this tool, they will write a simulation script with VerilogHDL, and logic with SFL. The uml2sfl was also developed by me in 2009. Since then, I extensively have upgraded the tools and methodology. 


\section{CURRENT DESIGN Flow OF THE PROJECTS}

Fig. 2 shows the current design flow of the projects which uses UML for specification and SystemC for verification. We are just in the first annual year since we start to use new method. Then we have still only a few reports with this method. But these reports shows good response on this methodology.

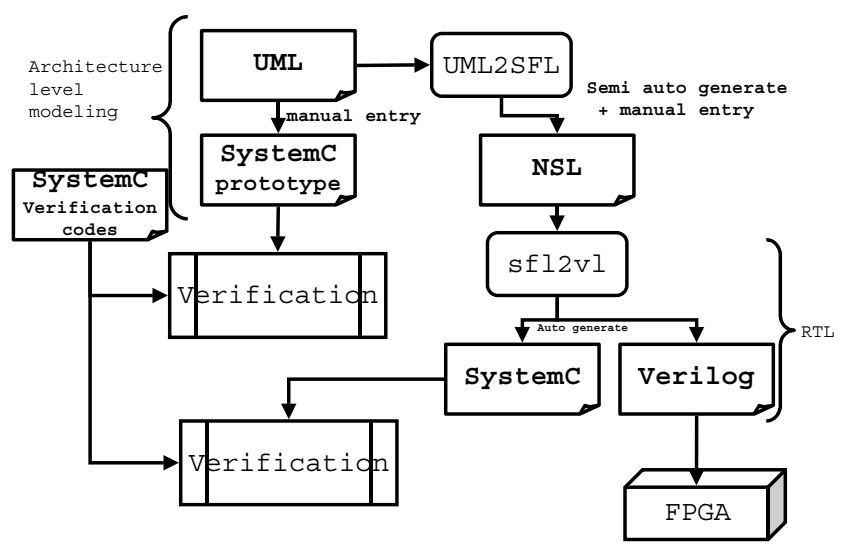

Fig. 2. Current projects design flow with UML and SystemC

The uml2sfl will convert UML class dialog to NSL or SFL. Where NSL is stand for the Next generation Synthesizable Language which is ancestor of SFL. SFL is the hardware description language for PARTHENON[13]. And the sfl2vl will convert them to RTL level VerilogHDL or RTL level SystemC.

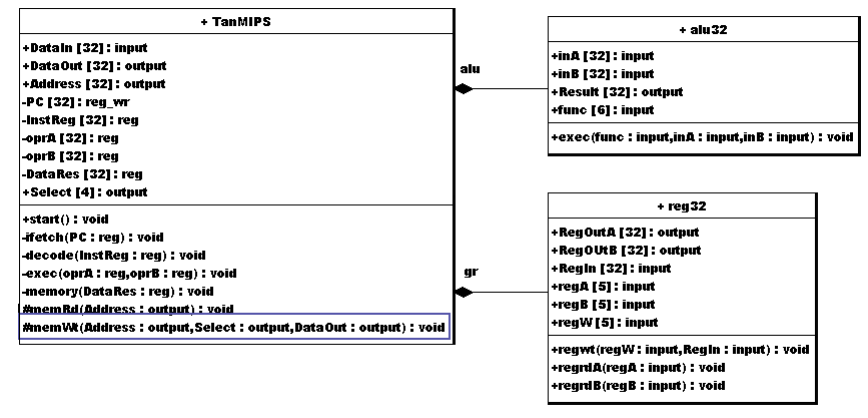

Fig. 3. UML Class Diagram for Multi-Cycle MIPS subset CPU

From 2009, we use UML class dialog for the initial design of our project. Fig. 3 is the dialog which I provided to students for their initial design. UML class dialog is consist of class name, attributes and operations. We map the class name to module name of hardware. We map the attributes to instances within the module such as registers, wires or input/output terminals. If visibility of a UML attribute is public, shown as '+', we assume that attribute will be a terminal which connect to outer modules. Or if visibility of the attribute is private shown as '-', we assume that attribute is placed inside the module.

Also we map the operations to functionality of the module. In SFL and NSL, we have special syntax to describe stage invocation functions and combinational functions. If visibility of an operation is public, shown as ' + ', we assume the operation will be invoked from other modules. In other words, the operation is incoming function to this module. Or if visibility of an operation is private, shown as '-', we assume the operation will be invoked within the module. The main usage of within module operation is pipeline stage or FSM state invocation. Or if visibility of an operation is protected, shown as '\#', we assume the operation will activate outer module function. In other words, the operation is outgoing function from this module.

In UML, black arrows means the composition relations. In this figure, two modules are imported to the main module as sub-modules. There is no example in the figure, but we can use white arrow as inheritance relations. In that case, all attributes and operations of parent class will be imported to the child class.

The uml2sfl will generate input/output terminals, registers, sub-module instances and stages with their arguments(pipeline registers). Therefore, it generates most of the static elements automatically, and students can concentrate on the behavior of each stages.

The Figure 4 is the operation part of the generated skeleton NSL code which is extracted from UML class diagram.

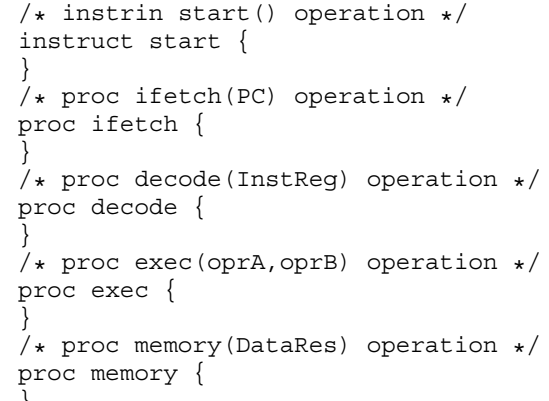

Fig. 4. The Operation Part of Generated NSL Skeleton Code Extracted From UML Class Diagram for Multi-Cycle MIPS subset CPU. The protected operations are not listed here, because they will be invoked from this module and there are no operations within this module.

The Figure 5 shows the modified code by the student for the ifetch and decode procedures. To save space, we only show a part of the CPU code.

\section{PREParation For undergraduate PROJeCtS}

Before we start undergraduate projects, we need a ton of preparations. Because the design methodology of many historical systems are obsolete, we must select or make alternative methodology. To select or make methodologies, I must consider a few problems.

1) Materials must be available legally for use in class room.

2) Tools should be freely available for students homework. 


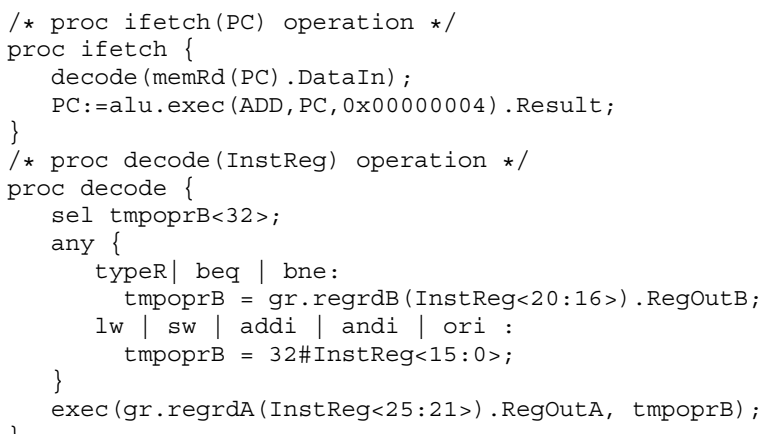

Fig. 5. Student Modification to the Extracted NSL

3) Complexity must be managed as low as possible.

4) Use of Hardware Description Languages.

\section{A. Hardware Design Environment}

The main hardware design language for the projects is NSL or SFL. We use VerilogHDL and/or SystemC for verification. And the target hardware is FPGA. The target device has been varied from time to time, depend on the availability and cost. The selection of the hardware description language is very important for the success of the projects. For short cutting the students installation, I compiled a set of these tools in an archive LiveCygwin.zip. Students can download it and extract it on some directory.

\section{B. REAL software}

Back to 1970 's, many computer engineers who are still active in this industry, read 'Lions' commentary on UNIX[16].' We were very excited with the detailed design of UNIX (the SOURCE) that is shown in Lions' book. His book was not publicly available until 1996. In addition to his book, Caldera put the license of ancient UNIX as open source in 2002[17].

Also, in 2003, Steve Wozniak said that Apple-I's schematics and ROM are public[18].

"The best anyone could say was that it was mine and that I made it public," Woz said to Briel.

Another major player in micro processor industry was Intel or Zilog with $\mathrm{CP} / \mathrm{M}$ for 8bit, and Intel with MS-DOS for 16bit CPU. In 2001, CP/M unofficial site's Gaby Chaudry negotiated with Lineo that was the license holder of $\mathrm{CP} / \mathrm{M}$, for publicly distribution of CP/M and related technology[19]. MS-DOS may not be open, but open source project FreeDOS became version 1.0 in 2006[20].

We also have Linux[21] and BSDs for advanced operating systems. I did use Linux for operating system projects, but I have not tried them for hardware projects, yet.

For students who love TV games, I contracted with Taito Corp. to use the Space Invader game software for laboratory education. Unfortunately, the program was binary form.

It is very important for us to get source code of our target projects. Because during the development of hardware we will have a lot of problems which are difficult to solve without source.

\section{Hardware documentations}

I have collected some hardware documentations by myself. I use these documents for our projects. It includes:

- MCS6500 Micro Computer Family Programming Manual[22]

- MCS-80/85 Family User's Manual[23]

- pdp11 processor handbook pdp11/20,15,r20[24]

- pdp11 processor handbook pdp11/45[25]

- pdp11 peripherals and interfacing handbook[26]

- MCS-86 USER'S MANUAL[27]

\section{Hardware design examples}

Students need to learn hardware description language. But it is not enough to design elegant system. In addition to learn the language, students must deeply read good design examples. For this purpose, I prepared some example circuits.

1) $\mathrm{SP} / 1$ : 8 bit 5 stage pipeline RISC (1998)

2) SN/X: 16bit non-pipeline RISC with compiler and assembler (2001)

3) my80: MCS-80 compatible CPU (2001)

4) m65: MCS6502 compatible CPU (2002)

5) mz80: Z80 compatible CPU (2002)

6) my88: i8088 semi-compatible but incomplete CPU (2002)

7) Stopwatch system with bitmap display on VGA

For all examples, I carefully design the data path to keep the number of gates as low as possible. Fortunately, many hardware manual in 70's show the block diagram of CPU. I checked the instruction set and data path repeatedly to minimize my design. Especially, for m65, I like 6502 and I know the instructions well, and I enjoyed to design it. The m65 CPU consist of two files one is CPU body, the other is ALU. The total number of the lines is about 1000 lines. It is not so difficult to read 1000 lines CPU. Therefore, I recommended students who are interesting in CPU design to read m65 deeply. The other students who were not interested in CPU design, used these example as is for their projects. Sometimes it had bugs and they repaired.

\section{UNDERGRADUATE PROJECTS}

\section{A. $C P / M 80$}

In this project, we decided to use solid state storage. We extended the address line of i8080A instruction compatible CPU up to 24 bits that is $16 \mathrm{MB}$ of address space. And we added new instructions to access extended address space which use three 8 bit registers to designate 24bit address. We also developed a special BIOS for this new storage system.

We used Altera EPF10K30AQC240-3 for the implementation. It uses 1260 logic cells and the maximum clock frequency is $8.9 \mathrm{MHz}$.

\section{B. Space Invaders}

This project also uses my80 as a CPU. But it incorporate many I/O functions and interrupt timer. Original Taito's Space Invaders had analog sound circuit based on OP amp 

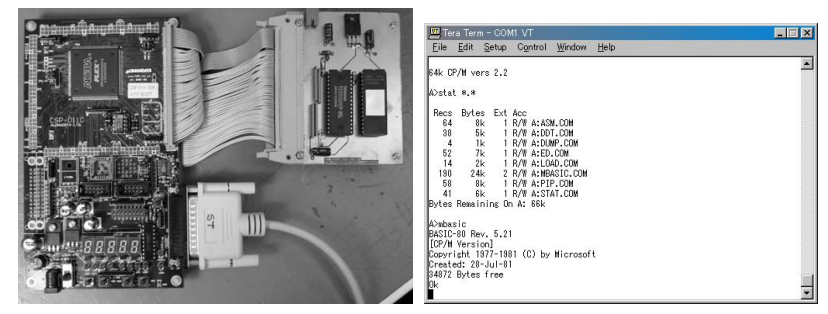

Fig. 6. The picture of CP/M running on Altera EPF10K30A

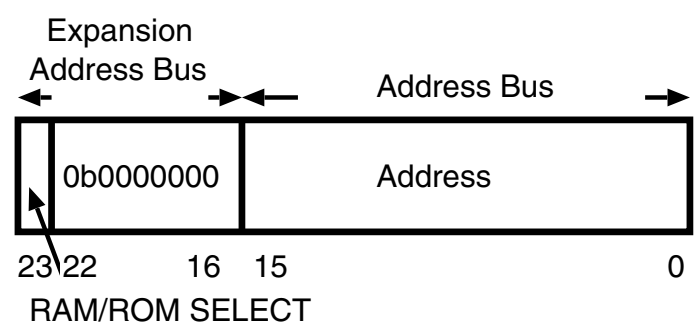

(a) Nomal Access Mode

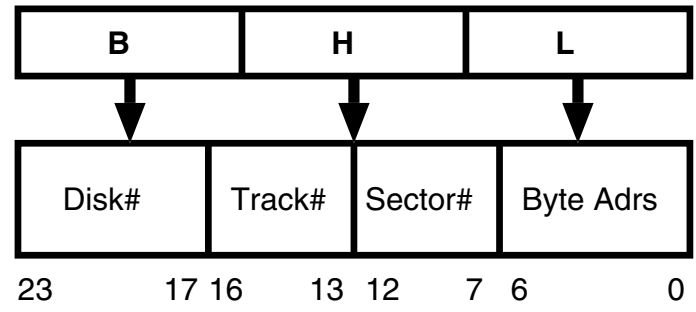

(b) RAM Disk Access Mode

Fig. 7. Address Assignment

and SN76477 sound chip. But we decided to make everything in digital with triangle wave generator, random noise generator and FIR filters. Also original Space Invaders had monochrome display and color tape attached on the screen. But we generate NTSC color signal with minimum analog circuit.
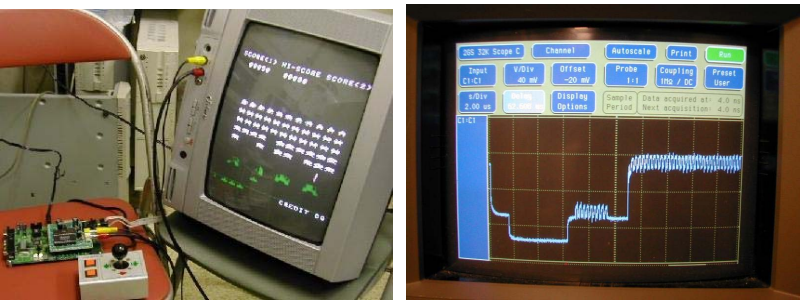

Fig. 8. Space Invaders compatible hardware running on Altera EPF10K30E and the NTSC signal from it

When we fitted into an FPGA (Altera's EPF10K30E-3), we used 1503 logic elements. It will work up to $20 \mathrm{MHz}$.

\section{PDP11}

The student for this project deeply read the m65 example. And he understand about addressing mode and it decoding

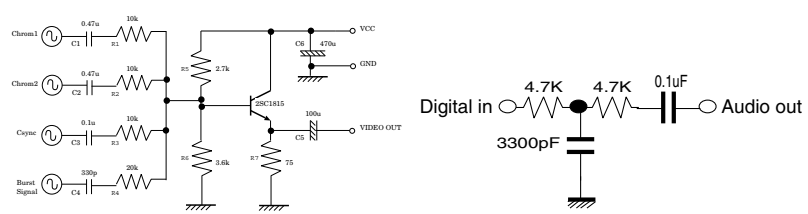

Fig. 9. The Analog part of Space Invader Project

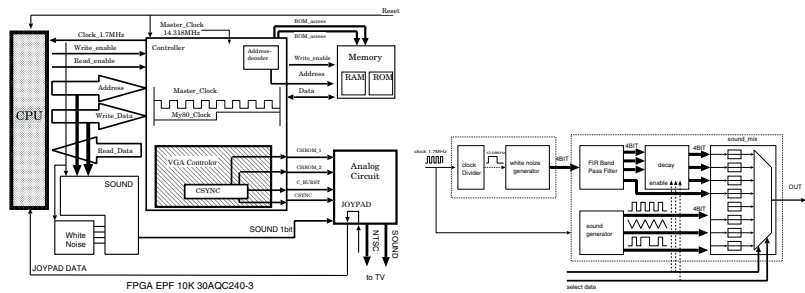

Fig. 10. The Block Diagram of Space Invader Project

method within CPU. We started to design CPU without MMU at first. While he developing CPU, I made a series of lectures on Lions Commentary on UNIX for him. There was an incomplete porting of GNU C compiler for PDP11, and we fixed the compiler to use in debugging our CPU. We also ported 'proc[28]' real time operating system to our CPU for demonstration of embedded system applications.

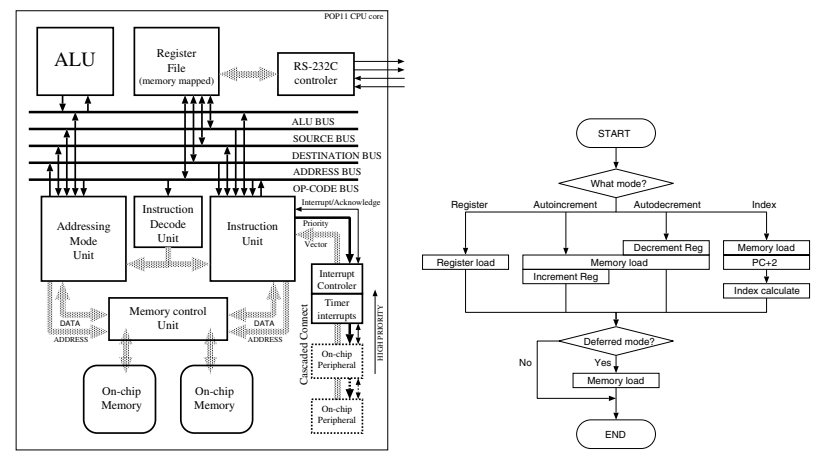

Fig. 11. The block diagram and addressing mode state chart of pdp11 compatible $\mathrm{CPU}$

As next step, we implemented MMU for our processor. And instead of old RK disk drive, we want to use IDE hard drive in our system. Then we made protocol converter from/to RK disk drive to/from IDE hard drive.

When implemented on an FPGA, pdp11 without MMU will use 1678 logic cells and it run up to $9 \mathrm{MHz}$ on Altera's EPF10K30E-3, and pdp11 with MMU will use 2687 logic cells and it run up to $20 \mathrm{MHz}$ on Altera's EP1K00-1.

\section{FreeDOS}

In this project, we tried to make i8086 instruction compatible CPU. It has many complex features such as prefix instruction, string instruction, segmentation, etc. We separated bus interface unit and execution unit of the CPU and implemented a instruction fetch queue as described in the MCS-86 manual. 


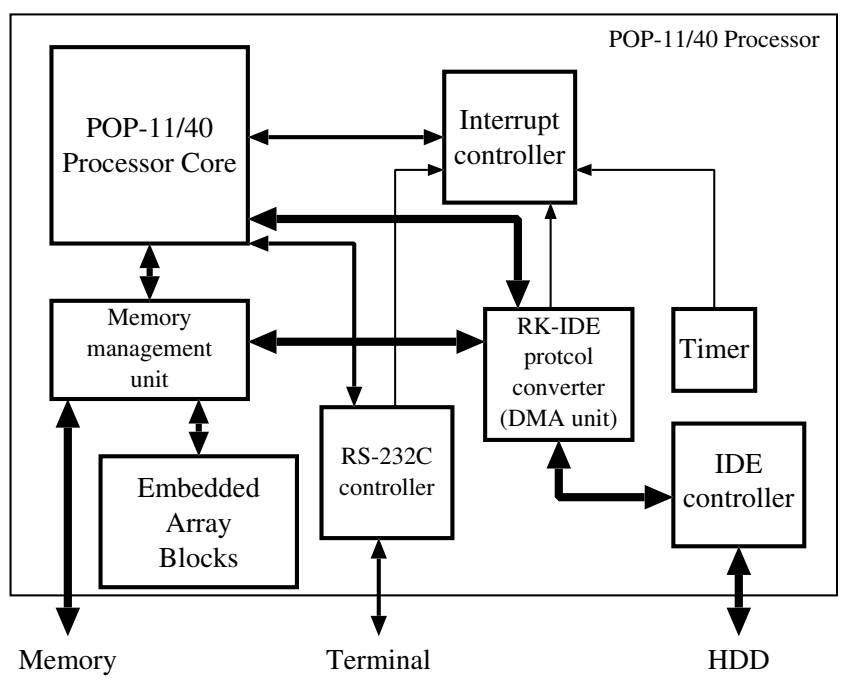

Fig. 12. The block diagram of pdp11 compatible CPU with MMU

When the CPU was ready to simulation, we ran the drystone benchmark on simulation. We also wrote BIOS routines which is compatible with PC. We implemented 6 bios entries: int 10, int 11, int 12, int 13, int 16, int 1a. At that time, FreeDOS requires 4 of i80186 instructions. Then we added these instruction to our processor.
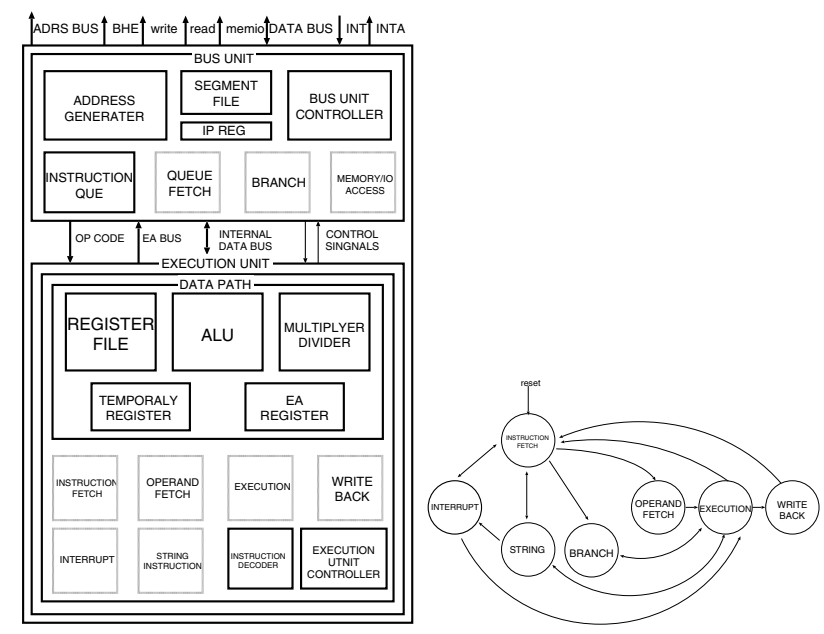

Fig. 13. The block diagram and state chart of i8086 compatible CPU running on Altera EP1K100

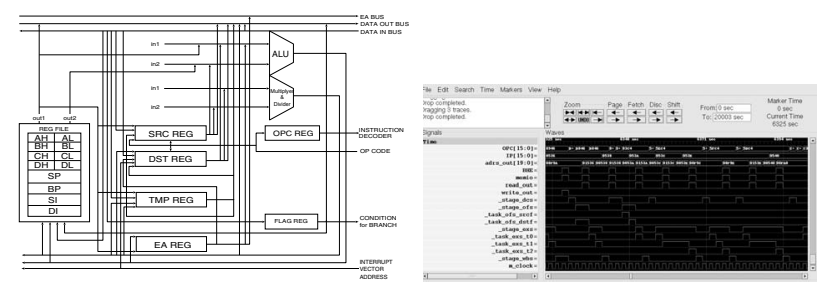

Fig. 14. The data path and timing chart of i8086 compatible CPU

When implemented in Altera FPGA EP1S10, the number of logic cells are 3884 and frequency is $9.16 \mathrm{MHz}$.

\section{E. Hardware Paint Tool}

This project did not use any CPU. All the controls are hard wired. The module will get input from PS/2 mouse and put picture on VGA display. The cursor is implemented as a sprite image.

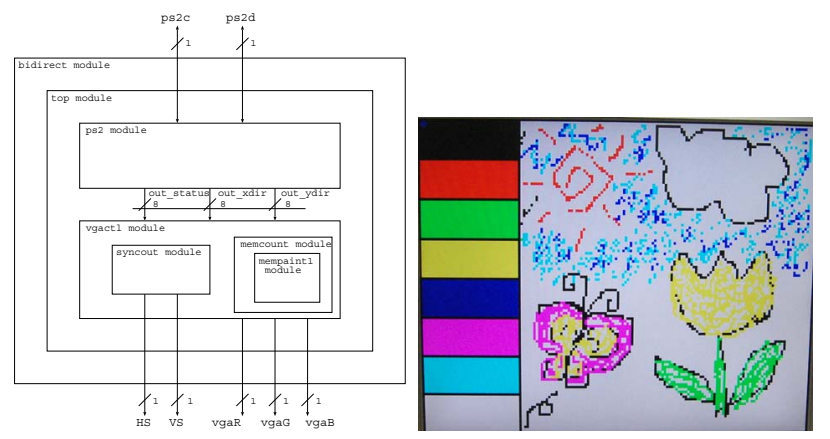

Fig. 15. Hardware Painting tool with Mouse running on Xilinx Spartan3 starter kit

\section{EXAMPLE PROJECT FOR SFL/NSL USERS}

We have two demonstrative project for SFL/NSL users. One is Apple-I software compatible system on an FPGA. We call it as AISoC. It includes 6502 compatible CPU, Apple-I software compatible keyboard and display interface which is converted to serial port, PS/2 keyboard interface, VGA character display interface, 7 segment LED interface, LCD panel interface, respectively. When complied for Spartan3

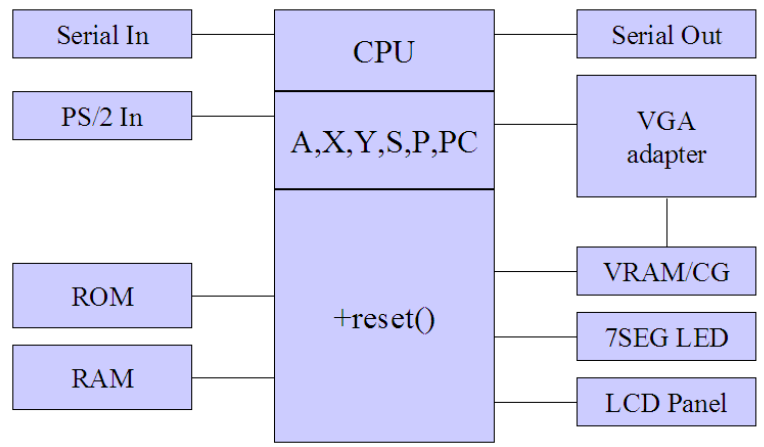

Fig. 16. Class Diagram of AISoC for Xilinx Spartan3/3E starter kit

xc3s200ft256-5, ISE reports that it will use 1208 LUTs and maximum clock frequency is $76.145 \mathrm{MHz}$. That means 70 times faster than original Apple-I. To be honest, we divided master clock by 2, then real CPU clock will be half of the report. Of course, it is still very fast compare to the original Apple-I.

Another example is Midway game platform which was used in Space Invaders. The students projects used NTSC video system for the display output. I changed the video system to VGA that is more usable for PC users. 


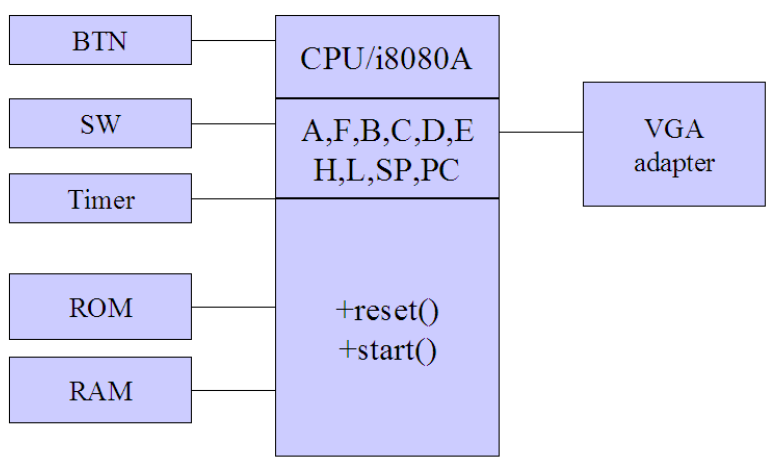

Fig. 17. Class Diagram of Midway Game Platform for Xilinx Spartan3/3E starter kit

When compiled for Spartan3 xc3s200ft256-5, ISE reports that it will use 1352 LUTs and maximum clock frequency is $241.074 \mathrm{MHz}$. We divided the clock by 5 then effective CPU clock is about $48 \mathrm{MHz}$. Original Midway game platform CPU was i8080A and it was worked about $2 \mathrm{MHz}$. In our example, the CPU run at one bus cycle as one CPU cycle where original i8080A takes 3 to 4 clocks. Therefore, we can assume that effective performance will be 70 to 90 times faster than original.

\section{CONCLUSION}

In this paper, I introduce my and my students project which reincarnate historic systems on FPGA. For these projects, students are required vast of knowledge such as compiler, operating system, bios, assembler, IO subsystems and of course CPU. The students who tried these projects were well educated. And many of them are in semiconductor industry.

And I introduced new methodology which utilize UML for hardware design. Instead of providing prototype CPU, I provide only UML class diagram for students. And they will make up for the behavior description of the CPU. During last semester, one student who had no experience on hardware description language tried the MIPS subset projects with UML. She began from learning hardware description language, but she took only three weeks to complete the project and ran a small program on simulation.

My example projects shows that with today's FPGA technology, historic systems will run many times faster than it was. With these advanced performance, I think, we are able to use historic system on FPGA not only for education but for embedded systems or other works.

\section{REFERENCES}

[1] ACM-IEEE. (2004) Acm report in the computing curricula: Computer engineering 2004. [Online]. Available: http://www.acm.org/education/education/curric_vols/CE-FinalReport.pdf

[2] C. Kon and N. Shimizu, "Development of 8 bit $\mathrm{cp} / \mathrm{m}$ machine with linux," Linux Conference Japan, 2001.

[3] H. Hayasaka, H. Haramiishi, and N. Shimizu, "Design of a pci bus interface on fpga," The 20th PARTHENON user meeting, 2001.
[4] Y. Iida and N. Shimizu, "Design of a pdp11 compatible cpu with programmable chip," 10th FPGA/PLD Design Conference, User presentation, pp. 93-100, 2002.

[5] — - "Design of pop-11(pdp-11 on programmable chip)," Proceedings of Asia and South Pacific Design Automation Conference 2004, pp. 571-572, 2004.

[6] Y. Iida, K. Yamaguchi, and N. Shimizu, "Invaders soc," The 22th PARTHENON user meeting, pp. 72-80, 2003.

[7] M. Oyama and N. Shimizu, "Development of i8086 instruction comaptible processor core and lsi development environment with sfl," 11th FPGA/PLD Design Conference, User presentation, pp. 35-41, 2003

[8] N. Kondo and N. Shimizu, "Invaders soc," The 25th PARTHENON user meeting, pp. 57-66, 2004

[9] S. Kinoshita, S. Namiki, M. Horiguchi, and N. Shimizu, "Development of paint tool hardware by learner and education effect," The 26th PARTHENON user meeting, pp. 11-17, 2005.

[10] H. Ohtsuki, S. Namiki, N. Shiozawa, K. Matsunaga, and N. Shimizu, "Applying mdd with uml for cpu development," The 31th PARTHENON user meeting, pp. 11-16, 2007.

[11] N. Shimizu, M. Ikura, W. Wiriya, and S. Chivapreecha, "A new logic circuit design methodology with uml," The 24th International Technical Conference on Circuits/Systems, Computers and Communications, 2009

[12] LIP6. (1990) Alliance vhdl. [Online]. Available: http://wwwasim.lip6.fr/recherche/alliance/

[13] Y. Nakamura, K. Oguri, H. Nakanishi, and R. Nomura, "An rtl behavioral description based logic design cad system with synthesis capability," IFIP Computer Hardware Description Language and their Applications, pp. 64-78, 1985.

[14] N. Shimizu, "The design of sfl2vl: Sfl to verilog convertor based on a lr-parser," Proceedings of the Workshop on Synthesis And System Integration of Mixed Information Technologies, pp. 251-257, 2003.

[15] M. Baxter. (2001) Open source in electronic design automation. [Online]. Available: http://www.linuxjournal.com/article/4428

[16] J. Lions, Lions' Commentary on UNIX. Charlottesville, VA, USA Peer-to-Peer Communications Inc., 1996.

[17] I. F. Darwin. (2002) Why caldera released unix: A brief history. [Online]. Available: http://linuxdevcenter.com/pub/a/linux/2002/02/28/caldera.html

[18] L. Kahney. (2003) Woz ok's apple i resurrection. [Online]. Available: http://www.wired.com/gadgets/mac/news/2003/09/60329

[19] T. Gasperson. (2001) $\mathrm{Cp} / \mathrm{m}$ collection is back online with an open source licence. [Online]. Available: http://www.theregister.co.uk/2001/11/26/cp_m_collection_is_back/

[20] FreeDOS. The freedos project. [Online]. Available: http://www.freedos.org

[21] M. McLagan. (1991) Linux online. [Online]. Available: http:/www.linux.org/

[22] MCS 6500 Microcomputer Family Programming Manual, MOS Technology Inc., Norristown, PA, USA, 1976.

[23] The MCS-80/85 Family Uuser's Manual, Intel, Santa Clara, CA, USA, 1986.

[24] pdp11/20,15,r20 processor handbook, digital equipment corporation, Maynard, MA, USA, 1972.

[25] pdp11/45 processor handbook, digital equipment corporation, Maynard, MA, USA, 1972.

[26] pdp11/peripherals and interfacing handbook, digital equipment corporation, Maynard, MA, USA, 1972.

[27] MCS-86 USER'S MANUAL, Intel, Santa Clara, CA, USA, 1979.

[28] proc real-time kernel. Nilsen Elektronikk as. [Online]. Available: http://www.nilsenelektronikk.no/ 